\title{
Análise da Produção bibliográfica internacional sobre Olivoturismo
}

\author{
Kim Wallner Kersanach \\ Vander Valduga ${ }^{\mathrm{b}}$
}

\section{Resumo}

Com crescimento no cultivo de oliveiras e o consequente aumento o consumo do azeite de oliva, inicia o interesse de consumidores por conhecer seu processo de produção. Essa curiosidade despertada em consumidores gera uma nova modalidade no turismo do país, o chamado olivoturismo, no qual, realizam-se atividades em torno do cultivo das oliveiras e a produção do azeite de oliva. Nos contextos acadêmico e comercial, o tema é um tanto recente e requer investigação, portanto, o problema para a presente pesquisa é: qual a situação atual (2019) da produção bibliográfica internacional sobre o olivoturismo? 0 presente trabalho se caracteriza como bibliográfico, exploratório, descritivo e qualitativo. 0 objetivo deste estudo é identificar a produção acadêmica existente em torno do assunto do olivoturismo no mundo. Foi identificado que esta atividade é ascendente e importante para o desenvolvimento rural, preservação cultural, entre outros aspectos. No âmbito acadêmico evidenciou-se a importância da realização de novas pesquisas, acompanhando o desenvolvimento de propriedades com atividade turística relacionada ao azeite de oliva.

Palavra-chave: Turismo no espaço rural; Agroturismo; Turismo Rural; Olivoturismo.

\begin{abstract}
Analysis of the International Bibliographic Production Olive Oil Tourism

With growth in the plantation of olive trees and the consequent increase in the olive oil, consumption increases the interest of consumers in knowing their production process. This increase in consumers curiosity generates a new modality of tourism in the country, the so-called olive oil tourism, in which activities are carried out around the plantation of olive trees and the production of olive oil. In the academic and commercial context, the theme is rather recent and needs to be investigated, so the present work question is: what is the current (2019) state of international bibliographical production on Olive oil tourism? This work is characterized as bibliographic, exploratory, descriptive and qualitative work on olive oil tourism. The objective of this study is to identify the academic production that exists around the subject of olive oil tourism in the world. It was identified that this activity is ascending and important for rural development, cultural preservation, among other aspects. In the academic context, the importance of conducting new research was evidenced, accompanying the development of properties with tourism activity related to olive oil.
\end{abstract}

Keywords: Tourism in rural space; Agrotourism; Rural Tourism; Olive Oil Tourism.

a. Bacharelado em Turismo na Universidade Federal do Paraná, Curitiba, Paraná, Brasil. E-mail: kim.kersanach@gmail.com

b. Pós-Doutorado em Hospitalidade na Universidade Anhembi Morumbi, São Paulo, São Paulo, Brasil. Docente no curso de Turismo na Universidade Federal do Paraná, Curitiba, Paraná, Brasil. E-mail: vandervalduga@gmail.com 


\section{Resumen}

\section{Olivoturismo: Un Análisis De La Producción Bibliográfica Internacional Sobre El Tema}

Con el crecimiento en el cultivo de olivos y el consiguiente aumento en el consumo de aceite de oliva, comienza el interés de los consumidores en su proceso de producción. Esta curiosidad suscitada en los consumidores genera una nueva modalidad en el turismo del país, el llamado oleoturismo, en el que se realizan actividades en torno al cultivo de olivos y la producción de aceite de oliva. En el contexto académico y comercial, el tema es bastante reciente y necesita ser investigado, por lo que el problema de la presente investigación es: ¿cuál es la situación actual (2019) de la producción bibliográfica internacional sobre oleoturismo? El presente trabajo se caracteriza por ser bibliográfico, exploratorio, descriptivo y cualitativo. El objetivo de este estudio es identificar la producción académica que existe en torno al tema del oleoturismo en el mundo. Se identificó que esta actividad es ascendente e importante para el desarrollo rural, la preservación cultural, entre otros aspectos. En el contexto académico, se evidenció la importancia de realizar nuevas investigaciones, que acompañen el desarrollo de propiedades con actividad turística relacionada con el aceite de oliva.

Palabras clave: Turismo en el espacio rural; Agroturismo; Turismo Rural; Oleoturismo

\section{INTRODUÇÃo}

Com registros de mais de 10.000 a.c., o cultivo da oliveira (Olea europaea) possui uma vasta história e cultura na qual permeia a produção do azeite. A partir dos elementos encontrados na elaboração do azeite, se pode iniciar um segmento dentro do turismo, o olivoturismo. 0 cultivo de oliveiras ocupa mundialmente cerca de 10 milhões de hectares, onde por volta de $90 \%$ dessa área está localizada na costa do Mediterrâneo (Coutinho et al., 2015). Segundo o Conselho Internacional do Azeite (Internacional Olive Concil - IOC, 2018), a Espanha desde 2000 vem liderando disparadamente a produção mundial de azeite, com um contínuo crescimento.

Neste contexto, a América Latina está representada pelos países: Argentina, Brasil, Chile e Uruguai. Em do território brasileiro, o cultivo da oliveira e a produção do azeite se faz presente nos Estados do Rio Grande do Sul, Paraná, São Paulo e Minas Gerais, (Marques, 2018). No Brasil existem 42 propriedades produtoras de azeites (Marques, 2018), que em 2018 produziram 58 mil litros de azeite. Existe uma estimativa de que chegue a 160 mil litros em 2019 (Scarton, 2019). Elias e Barbero (2017) destacam na Argentina a região denominada de Sudoeste Bonaerense "SOB", como uma área fértil e importante de cultivo de oliveiras para a produção de azeite de oliva. As autoras contabilizam 42 empresas localizadas apenas nesta região, enquanto Marques, 2018 estimava esta mesma quantidade em todo o Brasil.

A produção de azeite, assim como ocorre em outros produtos como o vinho e a cerveja, acaba gerando demanda de pessoas interessadas nos aspectos de sua produção, movimentando cadeias produtivas. Amador-Hidalgo (2017) destaca que o olivoturismo é uma maneira de auxiliar o desenvolvimento rural. Isto pode contribuir para que a população rural permaneça no local, já que com a inserção dessa atividade, o meio não dependerá apenas da produção agrícola. Desse modo, 
o olivoturismo parece ser um perfeito complemento para atividades de olivicultura, podendo ser uma alternativa econômica e um meio de preservação cultural.

As publicações acadêmicas têm como papel ajudar a compreender e subsidiar o desenvolvimento dessa atividade. Como primeiro passo é importante fazer um levantamento sobre a atividade para permitir o monitoramento de suas mudanças (Tregua et al., 2018). Ao mesmo tempo, é importante o mapeamento da produção científica para se compreender seus aspectos, abrangência, características e, no contexto científico, estabelecer aquilo que é chamado de estado da arte. Justifica-se a elaboração do presente trabalho em decorrência do Brasil, nos últimos 10 anos, ter ingressado efetivamente na produção de azeites de oliva. Os estados do Rio Grande do Sul e Minas Gerais destacam-se na produção de azeite. Outros estados, como o Paraná, apresentam ainda iniciativas tímidas. Neste cenário, o Rio Grande do Sul se apresenta como o maior produtor de azeite do país, representando $70 \%$ da produção nacional. A produção do estado concentra-se, principalmente, em municípios como Canguçu, Pinheiro Machado e Cachoeira do Sul.

A partir do crescimento da produção, ocorreu também o incremento no consumo, gerando interesse desses consumidores pelos aspectos produtivos, paisagens e demais características das regiões produtoras, convergindo para a criação do chamado olivoturismo. Trata-se de tema inovador no contexto acadêmico brasileiro do turismo e que requer atenção por estar ligado a aspectos rurais, produtivos, econômicos e sociais das regiões produtoras. Nesse contexto e a partir do problema anunciado, o objetivo central deste estudo é identificar e analisar a produção acadêmica existente sobre o olivoturismo no mundo e no Brasil até o presente (2019).

\section{OLIVOTURISMO: UMA ABORDAGEM INICIAL}

A pesquisa sobre o turismo baseado na produção de azeite de oliva apresenta diversas nomenclaturas como: Olivoturismo, Oleoturismo, Olive Tourism ou olive-based agritourism (Agüera et al., 2017). Encontra-se ainda as designações: "olive oil tourism" ou "olive oil based tourism"(Murgado, 2013). 0 termo surgiu inicialmente no ano de 2010 na Austrália (Alonso, 2010; Alonso e Northcote, 2010). Logo após, iniciaram-se os estudos no continente europeu com a publicação de artigos e livros direcionados a países com uma produção significativa de azeite, como Espanha e Itália (Millán-Vázquez et al.,2010; Millán-Vázquez et al., 2014b; De Salvo et al., 2013; Murgado, 2013). 0 olivoturismo se encontra no contexto das diversas atividades do turismo rural, enquadrando-se tanto como uma atividade passiva (paisagens e gastronomia), atividade ativa (atividades agrícolas) nas quais o turista pode ter participação prática. Atividades como visitas nos estabelecimentos de processamento de azeite, degustação de azeite, passeios guiados são algumas das práticas que podem ser encontradas no olivoturismo (Millán-Vázquez et al., 2010).

O olivoturismo é o conjunto de atividades que giram em torno do azeite e de todo seu processo de produção, desde as plantações até a extração e envase, da gastronomia local que leva o azeite como ingrediente, além de atividades envoltas com a natureza e a cultura que levam o turista a emergir 
na realidade do ambiente e território local (Murgado, 2013). Na Figura 1 são identificadas as experiências do olivoturismo em diversos níveis. Os níveis são representados distintamente por círculos: os círculos internos menores representam experiências de maior impacto nos turistas, com destaque às visitações nos centros de produção. As atividades representadas pelos maiores círculos e os mais externas, são responsáveis por causar menos impacto. Já a linha horizontal divide as atividades controláveis e não controláveis por parte das empresas turísticas.

Figura 1 - Componentes de uma experiência de Olivoturismo

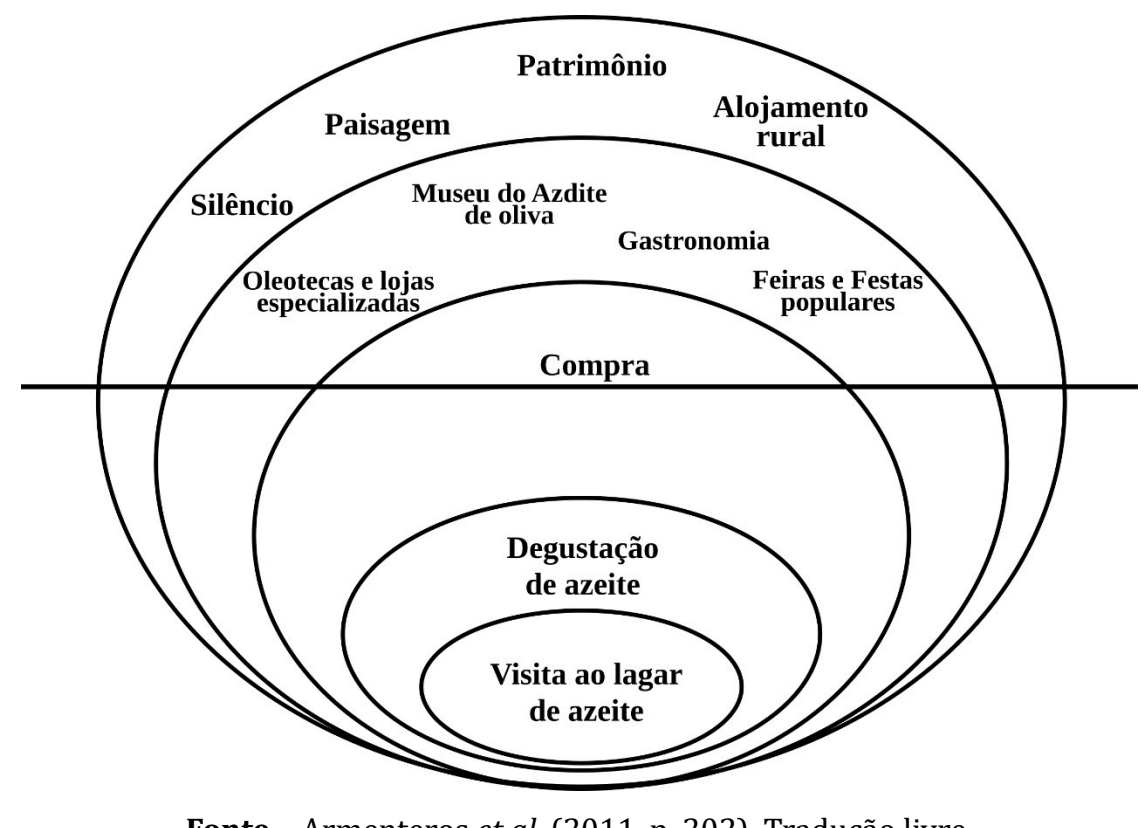

Fonte - Armenteros et al. (2011, p. 202). Tradução livre.

Uma divisão, pensada de acordo com a importância para a experiência do turista, é mostrada na Figura 1, onde a atração principal colocada pelo autor seria a visitação às plantações de oliveiras. Esse modelo de organização proposto visa a uma percepção em cima da qualidade do produto, assim como uma visão positiva do turismo por parte do produtor. Com base nas observações dos autores, é possível inferir que o olivoturismo faz parte de um turismo que envolve atividades culturais, industriais, rurais, arquitetônicas, gastronômicas, históricas, sociais, paisagísticas, entre outras.

Essas características da atividade permitem promover o azeite e seu patrimônio, viabilizando a promoção do produto, com agregação de elevação de valor e qualidade. Como consequência, o turismo que promove a qualidade do produto nas áreas rurais pode permitir a estimulação econômica local, evitando a migração de residentes para outras áreas, preservando-as e prevenindo o desemprego (Millán-Vázquez et al. 2014a). Neste âmbito, se pode afirmar que o olivoturismo contribui com a economia local, se tornando uma atividade complementar e uma fonte de renda alternativa. 


\section{METODOLOGIA}

A literatura foi pesquisada utilizando-se das bases de dados Redalyc ${ }^{1}$, Scopus ${ }^{2}$, Google Scholar ${ }^{3}$ e Periódicos CAPES ${ }^{4}$. As buscas nestas bases foram feitas com os seguintes termos: "Oleoturismo", "Olive Oil tourism" e "Oil-tourism", os mesmos foram definidos a partir de pesquisas realizadas online. Não foram estabelecidos limites temporais para a pesquisa. Essa pesquisa inicial identificou os termos como sendo os mais utilizados para a definição da atividade a ser analisada, além de todos se referirem a mesma atividade, porém em idiomas diferentes, como inglês e espanhol. 0 critério para a escolha das publicações foi o de ter relação direta com algum tema do contexto do olivoturismo. A primeira pesquisa foi realizada na plataforma Google Scholar e, a partir dela, iniciou-se o levantamento das publicações, que foram divididas em dois grupos: Grupo 1 Publicações existentes; Grupo 2 - Publicações coexistentes. Esta se referiu às publicações que apresentaram duplicidade nas plataformas, sendo, portanto, consideradas apenas uma vez.

0 presente trabalho se caracteriza como bibliográfico, exploratório, descritivo e qualitativo. Conforme exposto na introdução, o objetivo do trabalho é estabelecer um estado da arte sobre o tema olivoturismo. Estudos de estado da arte são caracterizados por ter como objetivo realizar um levantamento, sistematizando a produção da área de conhecimento a qual será analisada (Romanowski, 2006). Para obter uma visão geral e mais organizada, as publicações foram inseridas no programa Zotero 5.0.665, um software livre que auxilia na organização de coleções de documentos e gerações de citações e bibliografia. Este programa também permite a construção de uma linha do tempo contendo todas as publicações encontradas sobre o assunto pesquisado.

Além da linha do tempo, foram analisadas também a distribuição das publicações entre revistas da área (Quadro 2). Após as primeiras organizações, foram elaborados meios de análise visual que abordam questões como a relação quantidade de artigos/revistas, assuntos e palavras-chave. As classificações realizadas serviram para filtrar o tema e obter publicações mais específicas, além de organizar o contexto e permitir uma qualificação maior da análise qualitativa. Foram considerados, no levantamento, apenas artigos publicados em periódicos especializados, com o assunto principal de olivoturismo. Para a identificação das palavras e termos mais utilizados nas pesquisas foi adotado o programa online "Word it out", que elabora uma "nuvem" de palavras a partir

1. REDALYC. O projeto "Redalyc" (Rede de Revistas Científicas da América Latina e Caribe, Espanha e Portugal) é uma base de dados bibliográfica e de uma biblioteca digital de revistas de Acesso Aberto, suportado pela Universidade Autônoma do Estado do México com a ajuda de numerosas outras instituições de ensino superior e dos sistemas de informação. Disponível em: https://www.redalyc. org/. Acesso em 7 mai. 2019

2. SCOPUS. Disponível em: SciVerse, Scopus é um banco de dados de resumos e citações de artigos para jornais/revistas acadêmicos nos campos: científico, técnico, e de ciências médicas e sociais (incluindo as artes e humanidades). Acesso em7 mai. 2019.

3. GOOGLE SCHOLAR.O Google Scholar é um mecanismo de pesquisa na web de acesso livre que indexa o texto completo ou os metadados da literatura acadêmica em uma variedade de formatos e disciplinas de publicação Disponível em: Acesso em 7 mai. 2019

4. PERIÓDICOS CAPES. O Portal de Periódicos Capes, é uma biblioteca virtual que reúne e disponibiliza a instituições de ensino e pesquisa no Brasil produção científica internacional e nacional. Disponível em: https://www.periodicos.capes.gov.br/. Acesso em 7 mai. 2019. 
do texto. Com o qual foram construídas duas nuvens para a visualização dos assuntos e temas abordados e relacionados nas pesquisas sobre olivoturismo. A primeira "nuvem" foi elaborada a partir das palavras-chave dos artigos, já na segunda foram inseridos os resumos. A incidência das palavras e assuntos é representada de forma visual, onde, estas quando mais ocorrentes aparecem em maior escala. Para a nuvem dos assuntos abordados, foram excluídos conectores de texto e palavras que não apresentaram repetições, considerando apenas palavras com duas ou mais ocorrências.

\section{RESULTADOS E DISCUSSÃo}

A partir da identificação das publicações, foram elaborados quadros explicativos para identificar os autores, ano de publicação, revistas, abordagens, entre outras características. Deste modo, por meio do estudo exploratório foi possível visualizar a situação atual da investigação científica sobre o tema. 0 Quadro 1 se refere às bases de dados utilizadas para fazer o levantamento dos artigos e expões os artigos selecionados.

Quadro 1 - Publicações selecionadas para pesquisa

\begin{tabular}{|l|c|c|c|}
\hline Bases de Dados & Grupo 1 & Grupo 2 & Total \\
\hline Google Scholar & 16 & 0 & 16 \\
\hline Periódico CAPES & 12 & 2 & 10 \\
\hline Redalyc & 6 & 1 & 5 \\
\hline Scopus & 6 & 0 & 6 \\
\hline
\end{tabular}

Grupo 1- Publicações existentes; Grupo 2- Publicações coexistentes; Total- Total de publicações únicas.

Fonte - Elaborado pelos autores.

O Quadro 1 permite uma visualização da quantidade de publicações encontradas. Entre 2010 e 2019, foram identificadas um total de 40 publicações, onde três foram encontradas replicadas, evidenciando a reduzida produção acadêmica sobre o assunto. Além da linha do tempo, foi elaborado um quadro que sintetiza a produção científica sobre o tema por periódico (Quadro 2).

Quadro 2 - Periódicos onde os artigos investigados foram publicados

\begin{tabular}{|l|c|c|l|}
\hline Título do Periódico & \multicolumn{1}{|c|}{ ISSN } & $\begin{array}{c}\text { Quant. de } \\
\text { artigos }\end{array}$ & \multicolumn{1}{|c|}{ Autores das Publicações } \\
\hline Agricultural Sciences & $2156-8561$ & 1 & Millán-Vázquez, et al. (2014a) \\
\hline $\begin{array}{l}\text { Agriculture and } \\
\text { Human Values }\end{array}$ & $1572-8366$ & 1 & Northcote e Alonso (2011) \\
\hline $\begin{array}{l}\text { Boletín de la Asociación } \\
\text { de Geógrafos Españoles }\end{array}$ & $2605-3322$ & 1 & Millán-Vázquez, et al. (2014c) \\
\hline $\begin{array}{l}\text { British food journal } \\
\text { Cuadernos de } \\
\text { desarrollo rural }\end{array}$ & $0007-070 X$ & 1 & Alonso (2010) \\
\hline & $0122-1450$ & 2 & $\begin{array}{l}\text { Gutiérrez et al. (2011); } \\
\text { Millán-Vázquez, et al. (2014b) }\end{array}$ \\
\hline
\end{tabular}


Quadro 2 - Continuação.

\begin{tabular}{|c|c|c|c|}
\hline Título do Periódico & ISSN & $\begin{array}{l}\text { Quant. de } \\
\text { artigos }\end{array}$ & Autores das Publicações \\
\hline Cuadernos de Turismo & $1989-4635$ & 2 & $\begin{array}{l}\text { Agüera et al. (2017); } \\
\text { Sánchez e Ortega (2011) }\end{array}$ \\
\hline $\begin{array}{l}\text { Estudios y Perspectivas } \\
\text { en Turismo }\end{array}$ & 0327-5841 & 1 & Agüera et al. (2018) \\
\hline $\begin{array}{l}\text { European Journal of Tourism, } \\
\text { Hospitality and Recreation }\end{array}$ & $2182-4916$ & 1 & $\begin{array}{l}\text { López-Guzmán e } \\
\text { González-Fernández (2011) }\end{array}$ \\
\hline $\begin{array}{l}\text { Global Conference on } \\
\text { Business and Finance } \\
\text { Proceedings }\end{array}$ & 1941-9589 & 1 & Quesada et al. (2010) \\
\hline $\begin{array}{l}\text { International Journal of } \\
\text { Scientific Management } \\
\text { Tourism }\end{array}$ & $2386-8570$ & 1 & Mogollón et al. (2016) \\
\hline $\begin{array}{l}\text { International Journal of } \\
\text { Tourism Research }\end{array}$ & $1522-1970$ & 1 & Alonso e Northcote (2010) \\
\hline $\begin{array}{l}\text { International Journal } \\
\text { of World of Tourism }\end{array}$ & 2386-2319 & 2 & $\begin{array}{l}\text { Radhouance (2015); } \\
\text { Cuadra et al. (2014); }\end{array}$ \\
\hline Journal of Heritage Tourism & $1743-873 X$ & 1 & Alonso e Krajsic (2013) \\
\hline Mundo Agrario & $1515-5994$ & 1 & Morales e Gutiérrez (2010) \\
\hline $\begin{array}{l}\text { Olivae - revista oficial } \\
\text { del Consejo Oleícola } \\
\text { Internacional }\end{array}$ & 0255-996X & 1 & Guerra (2011) \\
\hline Panorama Socioeconomico & 0716-1921 & 1 & Millán-Vázquez, et al. (2010) \\
\hline Papers de turisme & $2255-1638$ & 1 & Guerra et al. (2014) \\
\hline $\begin{array}{l}\text { PASOS - Revista de Turismo } \\
\text { y Patrimonio Cultural }\end{array}$ & $1695-7121$ & 1 & Millán-Vázquez e Gutiérrez (2010) \\
\hline Revista de Ciencias Sociales & $1315-9518$ & 1 & Moreno et al. (2011) \\
\hline $\begin{array}{l}\text { Revista de Estudios } \\
\text { Regionales }\end{array}$ & 0213-7585 & 1 & Morales et al. (2015) \\
\hline Revista de Fomento Social & 00156043 & 1 & Amador-Hidalgo (2017) \\
\hline $\begin{array}{l}\text { Revista de Geografía Norte } \\
\text { Grande }\end{array}$ & 0379-8682 & 1 & Millán-Vázquez, et al. (2015) \\
\hline Revista Espacios & 0798-1015 & 1 & Cuadra et al. (2017) \\
\hline $\begin{array}{l}\text { Revista Interamericana de } \\
\text { Ambiente y Turismo }\end{array}$ & 0718-235X & 1 & Elias e Barbero (2017) \\
\hline $\begin{array}{l}\text { Revista Turismo e } \\
\text { Desenvolvimento }\end{array}$ & $1519-4744$ & 2 & $\begin{array}{l}\text { Campón-Cerro et al. (2014); } \\
\text { Millán-Vázquez, et al. (2010) }\end{array}$ \\
\hline Rural Society & 2204-0536 & 1 & Rodríguez- Cohard et al. (2017) \\
\hline Sustainability & $2071-1050$ & 3 & $\begin{array}{l}\text { Campón-Cerro et al. (2017); } \\
\text { Millán-Vázquez, et al. (2018); } \\
\text { Tregua et al. (2018) }\end{array}$ \\
\hline $\begin{array}{l}\text { Tourism and Hospitality } \\
\text { Management }\end{array}$ & $2372-5133$ & 3 & $\begin{array}{l}\text { De Salvo et al. (2013); } \\
\text { Hernández-Mogollón et al. (2019); } \\
\text { López- Guzmán et al. (2016) }\end{array}$ \\
\hline
\end{tabular}


Quadro 2 - Continuação.

\begin{tabular}{|l|c|c|l|}
\hline Título do Periódico & ISSN & $\begin{array}{c}\text { Quant. de } \\
\text { artigos }\end{array}$ & Autores das Publicações \\
\hline $\begin{array}{l}\text { Tourism Management } \\
\text { Perspectives }\end{array}$ & $2211-9736$ & 1 & $\begin{array}{l}\text { Arjona-Fuentes e } \\
\text { Amador-Hidalgo (2017) }\end{array}$ \\
\hline Total & & 37 & \\
\hline
\end{tabular}

Fonte - Elaborado pelos autores (2019).

Um total de 37 artigos distribuídos em 29 revistas foram publicados e considerados aqui. Diante disso, pode-se afirmar que as publicações encontradas a partir dos termos "oleoturismo", "olive oil tourism" e "oil-tourism" estão distribuídas, principalmente, entre os periódicos "Tourism and Hospitality Management" e "Sustainability". É possível observar que as revistas que dispõem da maior quantidade de publicações são espanholas, país que tem a maior produção de azeites do mundo. Em relação aos autores, foram identificados aqueles que possuem a maior quantidade de publicações e suas devidas instituições, são eles: Maria Genoveva Millán-Vázquez de la Torre da Universidad Loyola Andalucía e José Manuel Hernández-Mogollón da Universidad de Extremadura, ambos de universidades Espanholas. A partir do filtro aplicado, realizou-se uma linha do tempo da produção científica sobre o assunto para que seja possível obter um panorama do desenvolvimento científico do olivoturismo no mundo. A linha do tempo da Figura 2 foi dividida em anos, devido à quantidade de publicações existentes.

Figura 2 - Linha do tempo dos artigos selecionados

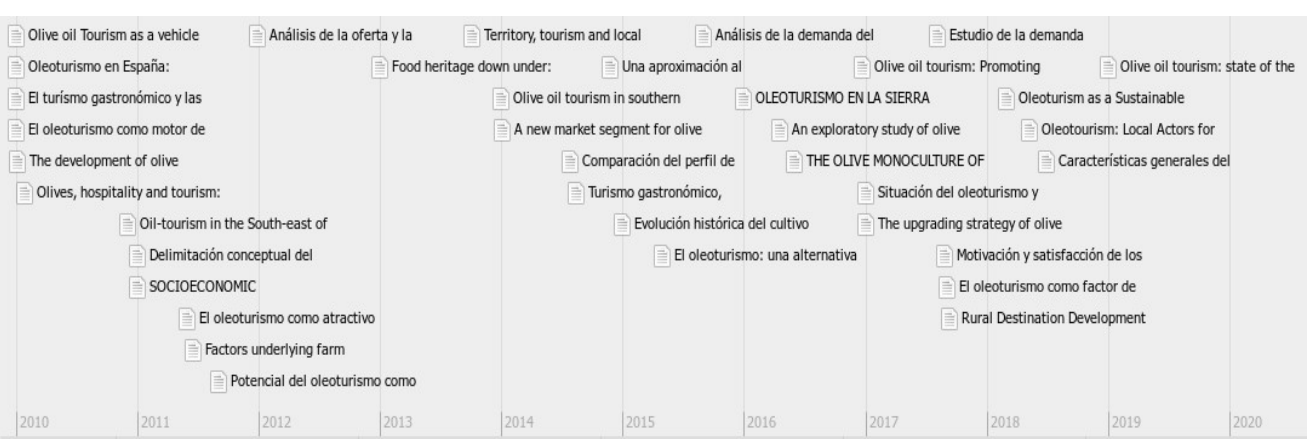

Fonte - Elaborado pelos autores a partir do sistema Zotero 5.0.66 (2019).

A partir da linha do tempo da Figura 2, pode-se perceber que os estudos em torno da atividade são recentes e a primeira publicação foi realizada no ano de 2010. Durante o período analisado (2010-2019), pode-se destacar os anos de 2010 e 2017 como sendo os anos com maiores quantidades de publicações. Já em 2012, 2013 e 2018 aparecem com um menor surgimento de artigos publicados. A Figura 3 foi confeccionada a partir da inserção de todas palavras-chaves encontradas nos artigos sobre olivoturismo. Esta, permite uma visualização mais clara dos assuntos abordados e segmentos utilizados na construção das pesquisas. Esta é constituída por 172 palavras chaves encontradas nos 36 artigos analisados e foi organizada de modo que as palavras maiores podem ser destacadas como as palavras que obtiveram maior frequência nos artigos e as menores a frequência foi menor. 
Figura 3 - Nuvem de palavras a partir das palavras-chave

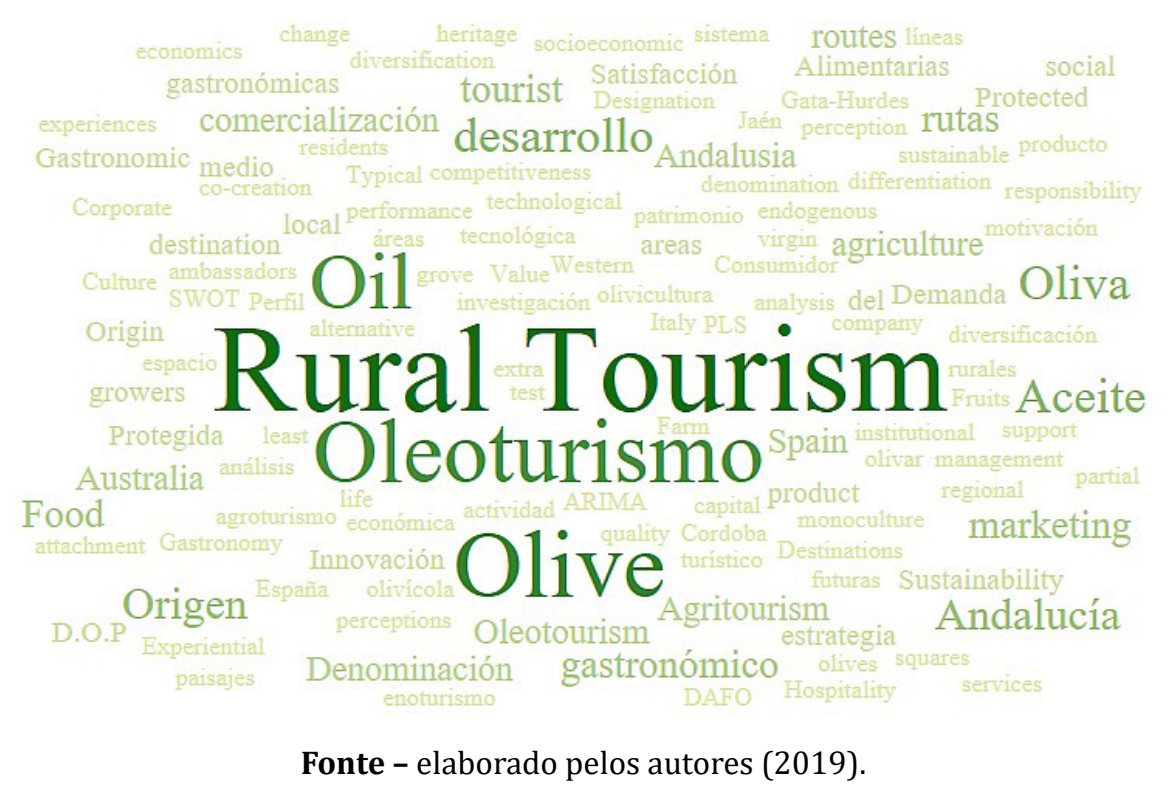

Na Figura 3, pode-se perceber que palavras como: "Rural Tourism", "olive", "oil", "oleoturismo", "desarrollo", são as mais utilizadas como palavras-chave entre as publicações analisadas. 0 esquema auxilia na identificação dos termos que publicações anteriores utilizam para abordar o assunto do Olivoturismo. Deste modo, foi possível perceber a importante relação que os autores estabelecem entre oleoturismo e turismo rural. Essa relação entre as duas atividades é apresentada em todos os artigos analisados. 0 olivoturismo é identificado como uma atividade única, podendo ser considerada uma iniciativa a prática do turismo rural sustentável e promotora da gastronomia (Millán-Vázquez et al. 2014a). 0 olivoturismo serve como complemento de outras tipologias turísticas, como o enoturismo ou o turismo rural (Morales et al., 2015). O olivoturismo faz parte, também, do turismo gastronômico, no qual vem se destacando como sendo uma das atividades de turismo rural mais importantes (Millán et al. 2014b).

A Figura 4 engloba os resumos dos artigos analisados a fim de demonstrar os temas mais abordados pelos autores. Dentre as 1416 palavras encontradas, foi realizado um filtro onde foram excluídos conectores de texto e palavras que não tinham repetição podendo apenas aparecer palavras citadas mais de 2 vezes, o que resultou no total de 295 palavras.

A Figura 4 apresentou como os principais assuntos das publicações os termos "olive”, "oliva”, “oil", turismo, "análisis", "Spain", "food", "findings", “areas", entre outras. Foi possível visualizar que muitas publicações citam, se referem, ou analisam áreas na Espanha, destacando-se como estudos de caso. As palavras mais destacadas e que apareceram em todos os artigos foram "olive", "oliva", turismo, "oil", também palavras principais dos artigos e que os definem. Pode-se destacar, também, que os artigos tratam o assunto como uma descoberta, um desenvolvimento, cultura, novas rotas, diversificação econômica. A partir das palavras-chave, também, foi possível identificar que as pesquisas tiveram principalmente o objetivo de analisar o setor, suas inclinações econômicas, paisagísticas, culturais e gastronômicas. 
Figura 4 - Componentes de uma experiência de Olivoturismo

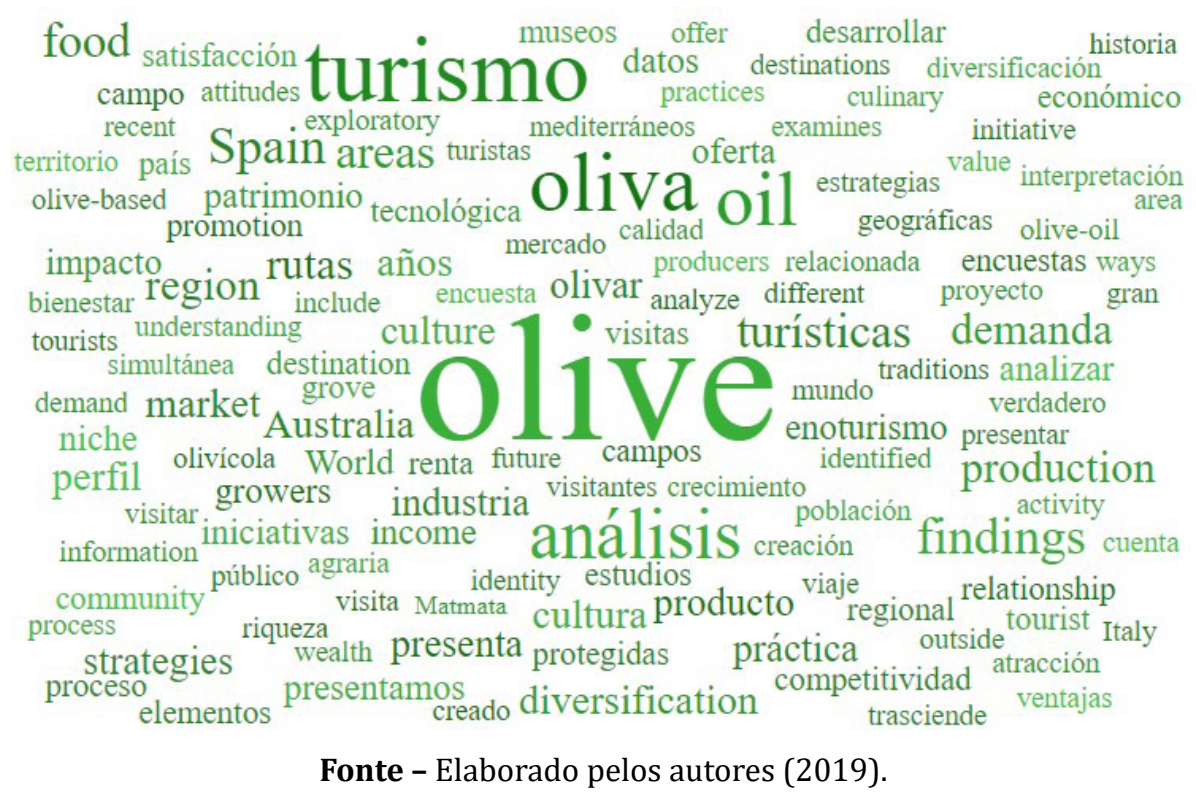

\section{CONCLUSÕES}

Devido ao olivoturismo ser uma atividade recente no mundo, estudos sobre ele atualmente (2019) são poucos. Foram identificados em um período de 9 anos, 37 artigos diretamente ligados ao tema. Estes artigos abordaram questões como a definição do segmento, atividades que podem e outras que já são desenvolvidas, locais que já desenvolvem perfis do turista do olivoturismo em regiões da Espanha, importância da atividade na zona rural e seus potenciais de desenvolvimento.

Pode-se destacar temas que podem ser explorados para pesquisas como preocupações com as motivações dos produtores em relação a prática do turismo em suas propriedades, a existência de propriedades que trabalham apenas com olivoturismo voltado a beleza e saúde, pesquisas sobre a divulgação do olivoturismo e o conhecimento do público sobre o assunto. Temas como impactos, dificuldades do desenvolvimento da atividade nas propriedades, formação de mão de obra, passos para se iniciar o turismo nas propriedades, ainda devem ser trabalhados e analisados a partir de diferentes perspectivas. Algumas questões para serem levantadas e estudadas futuramente em caráter sugestivo são: seria o olivoturismo uma atividade complementar a outros tipos de turismo? 0 olivoturismo tem potencial para se tornar um segmento principal no turismo?

No Brasil, a produção de trabalhos sobre o assunto é inexistente e o tema encontram-se em estado inicial. Atualmente, existem propriedades interessadas em pesquisa sobre o assunto, propriedades que já possuem projetos para o turismo e propriedades que já iniciaram as atividades turísticas. Portanto, se fazem necessários estudos mais detalhados para se acompanhar estes diversos estágios e entender sucesso e fracassos, para que os interessados possam implementar esta modalidade de turismo, facilitando o planejamento e o desenvolvimento da prática do olivoturismo. Sugere-se, portanto, que mais estudos sejam realizados a fim de subsidiar a atividade, seus produtores, integrando academia e os demais setores produtivos da sociedade. 


\section{REFERÊNCIAS}

Agüera, F. O., Cuadra, S. M., López-Guzmán, T., \& Morales, P. M. C. (2017). Estudio de la demanda existente en torno al oleoturismo. El caso de Andalucía. Cuadernos de Turismo, (39), 437-453.

Agüera, F. O., Cuadra, S. M., López-Guzmán, T., \& Morales, P. M. C. (2018). Características generales del oleoturismo y propuestas para futuras líneas de investigación. Estudios y perspectivas en turismo, 27(3), 487-505.

Alonso, A. D. (2010). Olives, hospitality and tourism: a Western Australian perspective. British Food Journal.

Alonso, A. D., \& Krajsic, V. (2013). Food heritage down under: olive growers as Mediterranean 'food ambassadors'. Journal of Heritage Tourism, 8(2-3), 158-171.

Alonso, A. D., \& Northcote, J. (2010). The development of olive tourism in Western Australia: A case study of an emerging tourism industry. International Journal of Tourism Research, 12(6), 696-708.

Arjona-Fuentes, J. M., \& Amador-Hidalgo, L. (2017). Olive oil tourism: Promoting rural development in Andalusia (Spain). Tourism Management Perspectives, 21, 100-108.

Armenteros, E. M. M., Ruíz, F. J. T., Rosa, M. P., \& Zamora, M. V. (2011). El aceite de oliva como elemento nuclear para el desarrollo del turismo. In Turismo Gastronómico: Estrategias de marketing y experiencias de éxito (pp. 191-220).

Campón-Cerro, A. M., Di-Clemente, E. L. I. D. E., Hernández-Mogollón, J. M., De Salvo, P. A. O. L. A., \& Calzati, V. (2014). Olive oil tourism in Southern Europe: Proposals for tourism development of olive grove rural areas. Turismo \& Desenvolvimento, 21(22), 63-73.

Campón-Cerro, A. M., Folgado-Fernández, J. A., \& Hernández-Mogollón, J. M. (2017). Rural destination development based on olive oil tourism: The impact of residents' community attachment and quality of life on their support for tourism development. Sustainability, 9(9), 1624.

Coutinho, E. F. (Ed.). (2015). Oliveira: aspectos técnicos e cultivo no sul do Brasil. Embrapa.

Cuadra, S. M., Cañero Morales, P., Orgaz Agüera, F., \& López Guzmán, T. (2014). Una aproximación al oleoturismo en Andalucía, España. International journal of world of tourism, 1 (2), 29-40.

Cuadra, S., M., Lopez-Guzman, T., Orgaz, F., \& Cañero, P. (2017). Motivación y satisfacción de los oleoturistas en España. El caso de Andalucía. Revista Espacios, 38(58).

De Salvo, P., Hernández-Mogollón, J. M., Di Clemente, E., \& Calzati, V. (2013). Territory, tourism and local products. The extra virgin oil's enhancement and promotion: a benchmarking Italy-Spain. Tourism and hospitality management, 19(1.), 23-34.

Elias, S. R., \& Barbero, A. C. (2017). Situación del oleoturismo y lineamientos para su desarrollo en la región del sudoeste bonaerense, Argentina. Revista interamericana de ambiente y turismo, 13(1), 91-104.

Guerra, I. R. (2011). Delimitación conceptual del aceite de oliva como recurso turístico. Olivae: revista oficial del Consejo Oleícola Internacional, (115), 32-47.

Guerra, I. R., Moreno, V. M., \& López, V. M. M. (2014). El oleoturismo como atractivo turístico en el medio rural español. Papers de turisme, (49-50), 89-103.

Gutiérrez, E. M. A., \& Fernandez, E. M. (2011). Análisis de la oferta y la demanda de oleoturismo en el sur de España: un estudio de caso. Cuadernos de Desarrollo Rural, 8(67), 181-202. 
Hidalgo, L. A. (2017). El oleoturismo como factor de desarrollo en zonas rurales de Andalucía. Revista de Fomento Social, 707-716.

International Olive Oil Council (2018). Olive Oil Production Leaders. Recuperado em: $<$ https://www.oliveoiltimes.com/pt/olive-oil-production-data>. Acesso em: 09 junho 2020.

López-Guzmán, T., Cañero Morales, P. M., Cuadra, S. M., \& Orgaz-Agüera, F. (2016). An exploratory study of olive tourism consumers. Tourism and hospitality management, 22(1), 57-68.

López-Guzmán, T., \& González-Fernández, V. (2011). Socioeconomic development in rural areas through the creation of tourist routes: An olive tourism approach in the Jaen province (Spain). European Journal of Tourism, Hospitality and Recreation, 2(2), 5-18.

Marques, S. (2018) 0 Guia de Azeites do Brasil: Safra 2018. São Paulo: Livrobits.

Millán-Vázquez., Agudo, E. M., \& Agudo, I. (2010). Oil-tourism in the South-east of Spain: The Necessity of Coordinating the Tourist Activity with the Agrarian one for the Development of the Rural Areas. Panorama socioeconómico, 28(41), 116-124.

Millán-Vázquez, Arjona-Fuentes, J. M., \& Amador, L. (2014a). A new market segment for olive oil: Olive oil tourism in the south of Spain. Agricultural Sciences, 2014a.

Millán-Vázquez, \& Agudo Gutiérrez, E. M. (2010). El turismo gastronómico y las Denominaciones de origen en el sur de España: Oleoturismo. Un estudio de caso.

Millán-Vázquez, \& Pérez, L. M. (2014b). Comparación del perfil de enoturistas y oleoturistas en España. Un estudio de caso. Cuadernos de Desarrollo Rural, 11(74), 167-188.

Millán-Vázquez., Fernández, E. M., \& Naranjo, L. M. P. (2014c). Turismo gastronómico, denominaciones de origen y desarrollo rural en Andalucía: situación actual. Boletín de la asociación de geógrafos españoles, (65).

Millán-Vázquez., Fernández, E. M. J., \& Pérez Guzman, L. (2010). Olive oil tourism as a vehicle for rural development in the province of Cordoba. Revista Turismo \& Desenvolvimento, 2(13), 59-68.

Millán-Vázquez, Hidalgo, L. A., \& Arjona-Fuentes, J. M. (2015). El oleoturismo: una alternativa para preservar los paisajes del olivar y promover el desarrollo rural y regional de Andalucía (España). Revista de Geografía Norte Grande, (60), 195-214.

Millán-Vázquez, Pablo-Romero, M. D. P., \& Sánchez-Rivas, J. (2018). Oleotourism as a sustainable product: an analysis of its demand in the south of Spain (Andalusia). Sustainability, 10(1), 101.

Mogollón, J. M. H., Di-Clemente, E., Folgado-Fernández, J. A., \& Campón-Cerro, A. M. (2019). Olive oil tourism: state of the art. Tourism and hospitality management, 25(1), 1-29.

Mogollón, J. M. H., Fernández, J. A. F., \& Cerro, A. M. C. (2016). Oleoturismo en la Sierra de Gata y las Hurdes (Cáceres): análisis de su potencial a través de un test de producto. International journal of scientific management and tourism, 2(1), 333-354.

Morales, E. F., \& Gutiérrez, E. M. A. (2010). El oleoturismo como motor de desarrollo rural: La denominación de origen de montoro-adamuz. Mundo Agrario: Revista de estudios rurales, 11(21), 14-27.

Morales, P. M. C., Guzmán, T. J. L. G., Cuadra, S. M., \& Agüera, F. O. (2015). Análisis de la demanda del oleoturismo en Andalucía. Revista de Estudios Regionales, (104), 133-149

Moreno, V. M., Rubio, J. M. Q., \& Guerra, I. R. (2011). Potencial del oleoturismo como diversificación económica del sector cooperativo agrario: el caso español. Revista de Ciencias Sociales, 17(3), 533-541. 
Murgado, E. M. (2013). Turning food into a gastronomic experience: olive oil tourism. Options Mediterranéennes, 106, 97-109.

Northcote, J., \& Alonso, A. D. (2011). Factors underlying farm diversification: the case of Western Australia's olive farmers. Agriculture and Human Values, 28(2), 237-246.

Quesada, J. M., Molina, V., \& Ruiz, I. (2010, May). Oleoturismo en España: Potencialidad de éxito internacional en escenarios actuales. In Global Conference on Business and Finance Proceedings (Vol. 5, No. 2, pp. 1534-1560).

Radhouance, C. (2015). Evolución histórica del cultivo del olivo, desarrollo local y oleoturismo. International Journal of World of Tourism, 2(4); 2-14.

Rodríguez-Cohard, J. C., Sánchez-Martínez, J. D., \& Gallego Simón, V. J. (2017). The upgrading strategy of olive oil producers in Southern Spain: origin, development and constraints. Rural Society, 26(1), 30-47.

Romanowski, J. P., \& Ens, R. T. (2006). As pesquisas denominadas do tipo" estado da arte" em educação. Revista diálogo educacional, 6(19), 37-50.

Sánchez, M., \& Ortega, R. (2016). The olive monoculture of Jaen: historical structure, heritage values and cultural-touristic importance. Cuadernos de Turismo, 37, 377-538.

Scarton, S. (2019). Produção de azeite extravirgem deste ano deve ser a maior do estado. Recuperado de: <https://estado.rs.gov.br/producao-de-azeite-extravirgem-deve-seramaior-do-rs-em-2019>. Acesso em: 20 maio 2019.

Tregua, M., D’Auria, A., \& Marano-Marcolini, C. (2018). Oleotourism: local actors for local tourism development. Sustainability, 10(5), 1492.

Recebido em: 04/06/2019

Aprovado em: 12/08/2019

\section{CONTRIBUIÇÃo}

Kim Wallner Kersanach: Definição do problema de pesquisa e objetivos; desenvolvimento da proposição teórica; realização da revisão bibliográfica e fundamentação teórica; coleta e análise de dados; elaboração de tabelas, gráficos e figuras; revisão crítica do manuscrito; redação do manuscrito e adequação do manuscrito às normas da RTA

Vander Valduga: Desenvolvimento da proposição teórica; realização da revisão bibliográfica e fundamentação teórica; escolha dos procedimentos metodológicos e revisão crítica do manuscrito 